\title{
A Comparative Study of Various Loss Functions in the Economic Tolerance Design
}

\author{
Jeh-Nan Pan \\ Department of Statistics \\ National Chen-Kung University, Tainan, Taiwan 70101, ROC \\ Jianbiao Pan \\ Department of Industrial and Manufacturing Engineering \\ California Polytechnic State University, San Luis Obispo, CA 93407, USA
}

\begin{abstract}
Engineering tolerance design plays an important role in modern manufacturing. In this paper, the Kapur's model was modified so that the economic specification limits for both symmetric and asymmetric losses can be established. Three different loss functions (1) Taguchi's quadratic loss function (2) Inverted Normal Loss Function (3) Revised Inverted Normal Loss Function are compared in the economic tolerance design. The relationships between the three loss functions and process capability indices for symmetric tolerance are established. The results suggest that the revised inverted normal loss function be used in determination of economic specification limits.
\end{abstract}

\section{INTRODUCTION}

Product quality is highly regarded in today's business environment. Generally speaking, quality characteristics can be divided into three types: nominal the best, larger the better and smaller the better. In the traditional concept of the quality evaluation system, a product is determined to be nonconforming if the quality characteristic of a product fails to meet the engineering specification limits and then a certain amount of quality loss is incurred. Reference [9], on the other hand, believed that a poorly designed product causes society to incur losses from the initial design stage to the product usage. Therefore, he defined the loss function as the deviation from the target/nominal quality characteristic. In other words, the Taguchi's quality loss is incurred when quality characteristics of a product deviates from its target value regardless how small the deviation is. Since then, the quality loss concept has been shifted from "defined by specification limits" to "defined by user" and Taguchi's loss function has been extensively used for determining the engineering tolerance.

The estimation of total quality losses plays an important role in the economic tolerance design. Reference [1] and [2] proposed an optimization method to develop the specification limits. The specification limits were determined on the basis of minimizing total cost or loss to the customer as well as to the producer. In the model, the scrap cost and rework cost were assumed to be the same. However, the scrap cost and rework cost may not be equal in many real manufacturing cases. For example, in metal cutting or machining processes, if a part is greater than the upper specification limit (USL), it still can be reworked to the specified length/thickness. However, if a part is less than the lower specification limit (LSL), it can no longer be used and has to be scrapped.

The objective of this study is to develop a new model of determining the economic specification limits. First, various loss functions were reviewed. The Kapur's model was then modified to relax the limitation that the scrap cost and the rework cost have to be same. The expected total losses including scrap, rework and inspection costs were estimated using three different loss functions: (1) Taguchi's quadratic loss function, (2) Inverted Normal Loss Function (INLF), and (3) Revised Inverted Normal Loss Function (RINLF). To decide which loss function is the best in the economic tolerance design, the relationships between process capability indices and expected loss per unit under normal distribution are derived. The results suggest RINLF be the most appropriate loss function in the economic tolerance design.

\section{LOSS FUNCTION REVIEW}

Taguchi [9] defined the quadratic loss function as

$$
L(y)=k(y-T)^{2}
$$

where $y$ is the quality characteristics, $k$ is the coefficient of quality loss. Taguchi's loss function has been extensively used for determining the engineering tolerance ([1]; [2]; [3]). The drawbacks of Taguchi's quality loss function are that it is unbounded and symmetrical ([4], [10]). In many manufacturing processes, it is unrealistic to assume the quality loss is unbounded even if the material, labor and other administrative costs are included. Asymmetric quality loss is also common in cases such as the scrap cost is different from the rework cost.

To overcome the unbounded loss in Taguchi's loss function, Reference [6] proposed a loss function as below:

$$
L(y)=\left\{\begin{array}{cll}
B(y-T)^{2} & \text { if } & y-T \leq \sqrt{K / B} \\
K & \text { if } & y-T>\sqrt{K / B}
\end{array}\right.
$$

where $K$ is the maximum value of quality loss, $B$ represents the coefficient of quality loss within the specification limits. In order to better describe the quality loss for the "Nominal the better" case of engineering specification, Reference [7] 
proposed inverted normal loss function (INLF) from a standpoint of normal probability density function (pdf). The INLF can be written as:

$$
L(y)=K\left\{1-\exp \left(-\frac{(y-T)^{2}}{2 \sigma_{L}^{2}}\right)\right\}
$$

where $K$ is the maximum loss if the characteristic deviated from the target, $\sigma_{L}^{2}$ is the parameter for controlling the shape of loss function depending on the realistic loss. In addition, Reference [7] and [8] proposed an asymmetric INLF as below:

$$
L(y)= \begin{cases}K_{1}\left\{1-\exp \left[-\frac{(y-T)^{2}}{2 \sigma_{L 1}^{2}}\right]\right\} & \text { if } \quad y<T \\ K_{2}\left\{1-\exp \left[-\frac{(y-T)^{2}}{2 \sigma_{L 2}^{2}}\right]\right\} & \text { if } \quad y \geq T\end{cases}
$$

Reference [5] proposed a revised inverted normal loss function (RINLF) to measure the quality loss for product interference. Due to the fact that quality loss will not incur when the quality characteristic falls within the neighborhood of target value from the customer's point of view, it would be more reasonable for a customer or manufacturer to specify an acceptable range $(\mathrm{L}, \mathrm{U})$ in which no quality loss would be incurred. Therefore, the Spiring's inverted normal loss function can be modified as (5).

$\mathrm{L}(\mathrm{y})=\left\{\begin{array}{cc}K_{1}\left\{1-\exp \left(-\frac{(y-L)^{2}}{2 \sigma_{L_{1}}^{2}}\right\}\right. & y<L \\ 0 & L \leq y \leq U \\ K_{2}\left\{1-\exp \left(-\frac{(y-U)^{2}}{2 \sigma_{L_{2}}^{2}}\right\}\right. & y>U\end{array}\right.$

where (L, U) is the acceptable range of a quality characteristic; $\mathrm{K}_{1}$ is the maximum loss if the characteristic deviates from the target and exceeds the LSL; $\mathrm{K}_{2}$ the maximum loss if the characteristic deviates from the target and exceeds the USL; $\sigma_{L_{1}}^{2}$ and $\sigma_{L_{2}}^{2}$ are the parameters for controlling the shape of function depending on the realistic loss.

\section{ECONOMIC TOLERANCE DESIGN}

To minimize total loss to the customer as well as to the manufacturer, Reference [1] and [2] proposed optimization models for determination of specification limits. Three costs were considered: inspection costs, scrap/rework costs, and loss due to variation. Assuming that both the target of a product $\mathrm{T}$ and the quality characteristic $\mathrm{Y}$ follow a normal distribution, Kapur's economic model of optimization can be written as:

$$
T C=L_{Q}^{*}+(1-q) \times S C+I C
$$

where TC is the total expected losses per unit product, $L_{Q}{ }^{*}$ is the expected loss per unit product shipped to the customer, SC is the scrap cost per unit, IC is the inspection cost per unit and $q$ is the fraction of good products actually shipped to the customer. In the model, the scrap cost and rework cost were assumed to be the same.

Considering that the quality losses above the upper or lower specification limit may not be equal, we revise Kapur's economic tolerance design model as

$$
T C=L^{*}+q_{1} \times S C+q_{2} \times R C+I C
$$

where $T C$ is total expected losses per unit product, $S C$ is the scrap cost per unit, $R C$ is the rework cost per unit, $I C$ is the inspection cost per unit, $L^{*}$ is the expected loss per unit product shipped to the customer using the above-mentioned loss functions, $q$ is the fraction of good products actually shipped to the customer and $q 1, q 2$ denotes the probability of scrap or rework respectively.

\section{ESTIMATION OF EXPECTED LOSS}

Next we will estimate the total expected loss per unit product based on the revised Kapur's economic tolerance design model. Assuming the quality characteristics $Y$ follows a normal distribution, i.e. $Y \sim N\left(\mu, \sigma^{2}\right)$, the expected loss per unit product is derived for revised Taguchi's quadratic, INIF, and RINLF loss functions.

As described in the introduction section, quality characteristics can be divided into three types: the nominal the best, the larger the best, and the small the best. The formulae of the total expected losses per unit product under two types of quality characteristics, i.e. the nominal the best (bilateral specification) and the smaller the better (unilateral specification) are derived. When the quality characteristics are the larger the better (unilateral specification), the derivation of total expected losses per unit product is very similar to the case of the smaller the better case. When the quality characteristics are the nominal the best (bilateral specification), we assume the lower specification limit $L S L=\mu-\eta_{1} \sigma \quad ; \quad$ the upper specification limit $U S L=\mu+\eta_{1} \sigma$; the product will be scrapped if $\mathrm{Y}$ exceeds the LSL and it can be reworked if $\mathrm{Y}$ exceeds the USL.

\section{A. Expected loss estimation using revised Taguchi's loss function}

Case 1: no inspection is performed. The total expected losses per unit product is the expected loss per unit product shipped to the customer, which can be written as

$$
\begin{aligned}
& L_{Q}=E\left[L_{Q}(Y)\right]=\int_{-\infty}^{\infty} L_{Q}(y) \times f(y) d y \\
& =k_{1}\left[\sigma^{2}+\{\mu-T\}^{2}\right] \\
& \quad+\left(k_{2}-k_{1}\right)\left\{\sigma(\mu-T) \phi\left(\frac{T-\mu}{\sigma}\right)+\left[(\mu-T)^{2}+\sigma^{2}\right]\left[1-\Phi\left(\frac{T-\mu}{\sigma}\right)\right]\right\}
\end{aligned}
$$

where $k_{1}, k_{2}$ represent the coefficient of two different 
quality losses.

Case 2: $100 \%$ inspection is performed. The expected loss per unit product shipped to the customer can be written as:

$$
\begin{aligned}
& L_{Q}^{*}=E\left[L_{Q}(Y)\right]=\int_{\mu-\eta_{1} \sigma}^{\mu+\eta_{2} \sigma} L_{Q}(y) \times f_{T}(y) d y \\
& =k_{1}\left[V\left(Y_{T}\right)+\left\{E\left(Y_{T}\right)-T\right\}^{2}\right] \\
& +\left(\frac{k_{2}-k_{1}}{q}\right)\left\{\left[(\mu-T)^{2}+\sigma^{2}\right]\left[\Phi\left(\eta_{2}\right)-\Phi\left(\frac{T-\mu}{\sigma}\right)\right]\right. \\
& \left.+\sigma(\mu-T) \phi\left(\frac{T-\mu}{\sigma}\right)-2 \sigma\left[(\mu-T)+\frac{\eta_{2}}{2}\right] \phi\left(\eta_{2}\right)\right\}
\end{aligned}
$$

where $E\left[Y_{T}\right]=\mu+\frac{\sigma}{q}\left[\phi\left(\eta_{1}\right)-\phi\left(\eta_{2}\right)\right]$ and

$$
V\left[Y_{T}\right]=\sigma^{2}\left\{1-\frac{\eta_{1}}{q} \phi\left(\eta_{1}\right)-\frac{\eta_{2}}{q} \phi\left(\eta_{2}\right)-\left[\frac{\phi\left(\eta_{1}\right)-\phi\left(\eta_{2}\right)}{q}\right]^{2}\right\}
$$

$q 1=1-\Phi\left(\eta_{1}\right) \quad q 2=1-\Phi\left(\eta_{2}\right) q=\Phi\left(\eta_{1}\right)+\Phi\left(\eta_{2}\right)-1$

$\phi(\bullet)$ and $\Phi(\bullet)$ denote the standard normal probability density function and the cumulative distribution. Note that $(\mu-T) / \sigma \leq \eta_{1} \leq \mu / \sigma$ and $\eta_{2} \geq(T-\mu) / \sigma$

Considering the scrap, rework, and inspection costs, the total expected loss per unit product can be calculated according to (7).

\section{B. Expected loss estimation using revised INLF}

Case 1: no inspection is performed. The total expected losses per unit product can be written as:

$$
\begin{aligned}
& L_{I N L F}=E\left[L_{I N L F}(y)\right]=\int_{-\infty}^{\infty} L_{I N L F}(y) \times f(y) d y \\
& =K_{1}\left\{\Phi\left(\frac{T-\mu}{\sigma}\right)-\frac{\sigma_{L 1}}{\sqrt{\sigma^{2}+\sigma_{L 1}^{2}}} \times \exp \left[-\frac{(\mu-T)^{2}}{2\left(\sigma^{2}+\sigma_{L 1}^{2}\right)}\right]\right. \\
& \left.\times \Phi\left(\frac{\sigma_{L 1}(T-\mu)}{\sigma \sqrt{\sigma^{2}+\sigma_{L 1}^{2}}}\right)\right\}+K_{2}\left\{1-\Phi\left(\frac{T-\mu}{\sigma}\right)\right. \\
& \left.-\frac{\sigma_{L 2}}{\sqrt{\sigma^{2}+\sigma_{L 2}^{2}}} \times \exp \left[-\frac{(\mu-T)^{2}}{2\left(\sigma^{2}+\sigma_{L 2}^{2}\right)}\right] \times\left[1-\Phi\left(\frac{\sigma_{L 2}(T-\mu)}{\sigma \sqrt{\sigma^{2}+\sigma_{L 2}^{2}}}\right)\right]\right\}
\end{aligned}
$$

Case 2: $100 \%$ inspection is performed. The expected loss per unit product shipped to the customer can be written as:

$$
\begin{aligned}
& L_{I N L F}^{*}=E\left[L_{I N L F}(Y)\right]=\int_{\mu-\eta_{1} \sigma}^{\mu+\eta_{2} \sigma} L_{I N L F}(y) \times f_{T}(y) d y \\
& =\frac{K_{1}}{q}\left\{\Phi\left(\frac{T-\mu}{\sigma}\right)+\Phi\left(\eta_{1}\right)-1-\frac{\sigma_{L 1}}{\sqrt{\sigma^{2}+\sigma_{L 1}^{2}}} \times\left\{\exp \left[-\frac{(\mu-T)^{2}}{2\left(\sigma^{2}+\sigma_{L 1}^{2}\right)}\right]\right\}\right. \\
& \left.\times\left[\Phi\left(\frac{\sigma_{L 1}(T-\mu)}{\sigma \sqrt{\sigma^{2}+\sigma_{L 1}^{2}}}\right)-\Phi\left(\frac{\sigma(\mu-T)-\eta_{1}\left(\sigma^{2}+\sigma_{L 1}^{2}\right)}{\sigma_{L 1} \sqrt{\sigma^{2}+\sigma_{L 1}^{2}}}\right)\right]\right\} \\
& +\frac{K_{2}}{q}\left\{\Phi\left(\eta_{2}\right)-\Phi\left(\frac{T-\mu}{\sigma}\right)-\frac{\sigma_{L 2}}{\sqrt{\sigma^{2}+\sigma_{L 2}^{2}}} \times\left\{\exp \left[-\frac{(\mu-T)^{2}}{2\left(\sigma^{2}+\sigma_{L 2}^{2}\right)}\right]\right\}\right. \\
& \left.\times\left[\Phi\left(\frac{\sigma(\mu-T)+\eta_{2}\left(\sigma^{2}+\sigma_{L 2}^{2}\right)}{\sigma_{L 2} \sqrt{\sigma^{2}+\sigma_{L 2}^{2}}}\right)-\Phi\left(\frac{\sigma_{L 2}(T-\mu)}{\sigma \sqrt{\sigma^{2}+\sigma_{L 2}^{2}}}\right)\right]\right\}
\end{aligned}
$$

Considering the scrap, rework, and inspection costs, the total expected loss per unit product can be calculated according to (7).

\section{C. $\quad$ Expected loss estimation using revised RINLF}

Case 1: no inspection is performed. The total expected losses per unit product can be written as

$$
\begin{aligned}
& L_{R I N L F}=E\left[L_{R I N L F}(y)\right]=\int_{-\infty}^{\infty} L_{R I N L F}(y) \times f(y) d y \\
= & K_{1}\left\{\Phi\left(\frac{L-\mu}{\sigma}\right)-\frac{\sigma_{L 1}}{\sqrt{\sigma^{2}+\sigma_{L 1}^{2}}}\right. \\
\times & \left.\exp \left[-\frac{(\mu-L)^{2}}{2\left(\sigma^{2}+\sigma_{L 1}^{2}\right)}\right] \times \Phi\left(\frac{\sigma_{L 1}(L-\mu)}{\sigma \sqrt{\sigma^{2}+\sigma_{L 1}^{2}}}\right)\right\} \\
+ & K_{2}\left\{1-\Phi\left(\frac{U-\mu}{\sigma}\right)-\frac{\sigma_{L 2}}{\sqrt{\sigma^{2}+\sigma_{L 2}^{2}}}\right. \\
\times & \left.\exp \left[-\frac{(\mu-U)^{2}}{2\left(\sigma^{2}+\sigma_{L 2}^{2}\right)}\right] \times\left[1-\Phi\left(\frac{\sigma_{L 2}(U-\mu)}{\sigma \sqrt{\sigma^{2}+\sigma_{L 2}^{2}}}\right)\right]\right\}
\end{aligned}
$$

Case 2: 100\% inspection is performed. The expected loss per unit product shipped to the customer can be written as:

$$
\begin{aligned}
& L_{\text {RINLF }}^{*}=E\left[L_{R I N L F}(Y)\right]=\int_{\mu-\eta_{1} \sigma}^{\mu+\eta_{2} \sigma} L_{R I N L F}(y) \times f_{T}(y) d y \\
& =\frac{K_{1}}{q}\left\{\Phi\left(\frac{L-\mu}{\sigma}\right)+\Phi\left(\eta_{1}\right)-1-\frac{\sigma_{L 1}}{\sqrt{\sigma^{2}+\sigma_{L 1}^{2}}} \times\left\{\exp \left[-\frac{(\mu-L)^{2}}{2\left(\sigma^{2}+\sigma_{L 1}^{2}\right)}\right]\right\}\right. \\
& \left.\times\left[\Phi\left(\frac{\sigma_{L 1}(L-\mu)}{\sigma \sqrt{\sigma^{2}+\sigma_{L 1}^{2}}}\right)-\Phi\left(\frac{\sigma(\mu-L)-\eta_{1}\left(\sigma^{2}+\sigma_{L 1}^{2}\right)}{\sigma_{L 1} \sqrt{\sigma^{2}+\sigma_{L 1}^{2}}}\right)\right]\right\} \\
& +\frac{K_{2}}{q}\left\{\Phi\left(\eta_{2}\right)-\Phi\left(\frac{U-\mu}{\sigma}\right)-\frac{\sigma_{L 2}}{\sqrt{\sigma^{2}+\sigma_{L 2}^{2}}} \times\left\{\exp \left[-\frac{(\mu-U)^{2}}{2\left(\sigma^{2}+\sigma_{L 2}^{2}\right)}\right]\right\}\right. \\
& \left.\times\left[\Phi\left(\frac{\sigma(\mu-U)+\eta_{2}\left(\sigma^{2}+\sigma_{L 2}^{2}\right)}{\sigma_{L 2} \sqrt{\sigma^{2}+\sigma_{L 2}^{2}}}\right)-\Phi\left(\frac{\sigma_{L 2}(U-\mu)}{\sigma \sqrt{\sigma^{2}+\sigma_{L 2}^{2}}}\right)\right]\right\}
\end{aligned}
$$

where $\eta_{1} \geq(\mu-L) / \sigma$ and $\eta_{2} \geq(U-\mu) / \sigma$

Considering the scrap, rework, and inspection costs, the total expected loss per unit product can be calculated according to (7). Estimation of the expected losses for unilateral specification can be derived similarly.

\section{RELATIONSHIP BETWEEN LOSS FUNCTION AND PROCESS CAPABILITY INDICES}

We have described expected loss estimation using Taguchi's quadratic, INLF, and RINLF loss functions. To answer which loss function is appropriate for determining the economic specification limits, it is necessary to explore the relationship between loss function and process capability indices.

Assuming that a quality characteristics Y follows a normal distribution, the process capability indices for a bilateral specification are: 


$$
\begin{aligned}
& C_{p}=\frac{U S L-L S L}{6 \sigma}=\frac{\Delta}{3 \sigma} \\
& C_{p k}=\min \left\{C_{p l}, C_{p u}\right\}=\min \left\{\frac{\mu-L S L}{3 \sigma}, \frac{U S L-\mu}{3 \sigma}\right\}=\frac{\Delta-|\mu-M|}{3 \sigma} \\
& C_{p m}=\frac{U S L-L S L}{6\left\{E(Y-T)^{2}\right\}^{\frac{1}{2}}}=\frac{\Delta}{3 \sqrt{\sigma^{2}+(\mu-T)^{2}}}
\end{aligned}
$$

where $M=\frac{U S L+L S L}{2}$ is the center of a bilateral specification and $\Delta=\frac{U S L-L S L}{2}$ denotes half of the specification width.

To explore the relationships between loss functions and process capability indices for symmetric tolerance, we first assume that quality characteristic of a symmetric tolerance ( $T$ $=M$ ), as shown in Fig. 1, follows a normal distribution. If the process capability indices $C_{p}$ and $C_{p k}$ are known, then one can obtain $|\mu-M|=3 \sigma\left(C_{p}-C_{p k}\right)$. Thus,

$$
\begin{aligned}
& L S L=\mu-\eta_{1} \sigma \Rightarrow \eta_{1}=[\Delta-(T-\mu)] / \sigma=\left[3 \sigma C_{p}-(T-\mu)\right] / \sigma \\
& U S L=\mu+\eta_{2} \sigma \Rightarrow \eta_{2}=\left[3 \sigma C_{p}+(T-\mu)\right] / \sigma
\end{aligned}
$$

When the process average $\mu$ is equal to the target value $T$, then $C_{p}=C_{p k}$ and $\eta_{1}=\eta_{2}=\eta=3 C_{p}$. Note that only the asymmetric loss $(\mathrm{SC} \neq \mathrm{RC})$ is plotted in Fig. 1 since the symmetric loss $(\mathrm{SC}=\mathrm{RC})$ can be considered as a special case of asymmetric loss. By utilizing $C_{p}$, one can derive the relationships between three different loss functions and process capability indices.

To explore the relationship between the revised Taguchi's loss function and process capability indices, we first assume the quality losses exceed the upper or lower specification limit are not equal and $k_{1}, k_{2}$ represent the coefficient of two different quality losses, namely, $k_{1}=K_{1} / \Delta^{2}=K_{1} /\left(3 \sigma C_{p}\right)^{2} ; k_{2}=K_{2} / \Delta^{2}=K_{2} /\left(3 \sigma C_{p}\right)^{2}$, where $K_{1}$ denotes the maximum loss if the quality characteristic deviates from the target and exceeds the LSL; and $K_{2}$ denotes the maximum loss if the characteristic deviates from the target and exceeds the USL. The expected loss per unit product shipped to the customer is

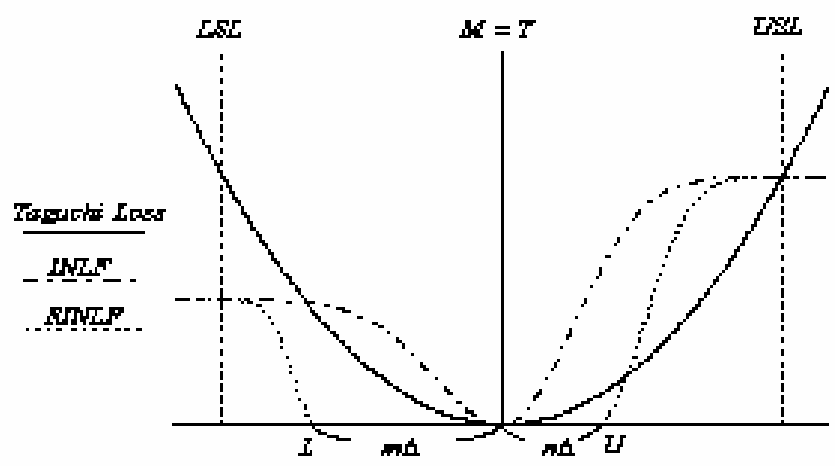

Fig. 1. Comparison of three loss functions for symmetric tolerance

$$
L_{Q}^{*}=\left(K_{1}+K_{2}\right)\left\{\frac{2 \Phi\left(3 C_{p}\right)-6 \phi\left(3 C_{p}\right) C_{p}-1}{18\left[2 \Phi\left(3 C_{p}\right)-1\right] C_{p}^{2}}\right\}
$$

Considering the scrap, rework and inspection costs, the total expected loss per unit product is

$$
T C=L_{Q}^{*}+\left[1-\Phi\left(3 C_{p}\right)\right] \times(S C+R C)+I C
$$

To explore the relationship between INLF and process capability indices and to achieve INLF's maximum loss at specification limits, we first set the parameters $\sigma_{L 1}=\sigma_{L 2}=\Delta / 4=[(3 \sigma) / 4] C_{p}$ according to the rule proposed by [7]. Then, the expected loss per unit product shipped to the customer is

$$
\begin{aligned}
L_{I N L F}^{*}= & \frac{\left(K_{1}+K_{2}\right)}{2 \Phi\left(3 C_{p}\right)-1} \times\left\{\Phi\left(3 C_{p}\right)-\frac{1}{2}\right. \\
& \left.-\frac{3 C_{p}}{\sqrt{16+9 C_{p}^{2}}}\left[\Phi\left(\sqrt{16+9 C_{p}^{2}}\right)-\frac{1}{2}\right]\right\}
\end{aligned}
$$

The total expected losses per unit product is $T C=L_{I N L F}^{*}+\left[1-\Phi\left(3 C_{p}\right)\right] \times(S C+R C)+I C$

To explore the relationship between RINLF and process capability indices and to achieve RINLF's maximum loss at specification limits, we first set the parameters: $\sigma_{L 1}=\frac{L-L S L}{4}=\frac{3 \sigma(1-m) C_{p}}{4} \sigma 2=\frac{U S L-L}{4}=\frac{3 \sigma(1-n) C_{p}}{4}$ according to the rule proposed by [7]. Then the expected loss per unit product shipped to the customer can be written as

$L_{R I N L F}^{*}=\frac{K_{1}}{2 \Phi\left(3 C_{p}\right)-1}\left\{\Phi\left(-3 m C_{p}\right)+\Phi\left(3 C_{p}\right)-1-\left\{\exp \left[-\frac{72 m^{2} C_{p}^{2}}{16+9(1-m)^{2} C_{p}^{2}}\right]\right\}\right.$

$\left.\times \frac{3(1-m) C_{p}}{\sqrt{16+9(1-m)^{2} C_{p}^{2}}} \times\left[\Phi\left(-\frac{9 m(1-m) C_{p}^{2}}{\sqrt{16+9(1-m)^{2} C_{p}^{2}}}\right)-\Phi\left(\frac{-16-9(1-m) C_{p}^{2}}{\sqrt{16+9(1-m)^{2} C_{p}^{2}}}\right)\right]\right\}$

$$
\begin{aligned}
& +\frac{K_{2}}{2 \Phi\left(3 C_{p}\right)-1} \times\left\{\Phi\left(3 C_{p}\right)-\Phi\left(3 n C_{p}\right)-\left\{\exp \left[-\frac{72 n^{2} C_{p}^{2}}{16+9(1-n)^{2} C_{p}^{2}}\right]\right\}\right. \\
& \left.\times \frac{3(1-n) C_{p}}{\sqrt{16+9(1-n)^{2} C_{p}^{2}}} \times\left[\Phi\left(-\frac{-16-9(1-n) C_{p}^{2}}{\sqrt{16+9(1-n)^{2} C_{p}^{2}}}\right)-\Phi\left(\frac{9 n(1-n) C_{p}^{2}}{\sqrt{16+9(1-n)^{2} C_{p}^{2}}}\right)\right]\right\}
\end{aligned}
$$

where $m=\frac{T-L}{\Delta}$ denotes the difference between the target and the lower limit for the acceptable range, in which no quality loss will be incurred, divided by half of the specification width; $n=\frac{U-T}{\Delta}$ denotes the difference between the target and the upper limit for the acceptable range, divided by half of the specification width. The total expected loss per unit product is $T C=L_{R I N L F}^{*}+\left[1-\Phi\left(3 C_{p}\right)\right] \times(S C+R C)+I C$

When a process average $\mu$ is greater or less than the target value $\mathrm{T}$, the derivation of the relationship between process 
capability indices and loss functions is similar to the case of process average $\mu=T$ as described above.

The relationship between loss function and process capability indices for asymmetric tolerance can be derived similarly.

VI. SELECTION OF THE APPROPRIATE LOSS FUNCTION FOR ECONOMIC TOLERANCE DESIGN

Based on the relationship between process capability indices and the total expected losses per unit product for various loss functions, the total expected losses per unit product for three loss functions under various $C_{p}$ can be compared. We have estimated the total expected losses per unit product under various $C_{p}$ for both the symmetric and asymmetric tolerances when a process average $\mu$ equals to $T$. Fig. 2 shows the total expected losses per unit product decline as the process capability index $C_{p}$ improves regardless which loss function is used (Here the case of symmetric tolerance and symmetric loss is illustrated as an example). Notice that the failure/defect rate is $66 \mathrm{ppm}$ (part per million) for $C_{p}=1.33$ or 4 sigma process, $0.54 \mathrm{ppm}$ for $C_{p}=1.67$ or 5 sigma process, and $0.002 \mathrm{ppm}$ for $C_{p}=2$ or 6 sigma process. When $C_{p}=2$, the total expected losses per unit product calculated using three different loss functions are $\$ 0.14\left(K_{1}+K_{2}\right)$ for the revised Taguchi's quadratic loss function, $\$ 0.084\left(K_{1}+K_{2}\right)$ for the INLF loss function, and $\$ 0.00004\left(K_{1}+K_{2}\right)$ for the RINLF, respectively. It appears that the expected loss per unit product calculated using RINLF is the most close to the failure/defect rate. Since engineering specification plays a key impact in the calculation of $C_{p}$ or $C_{p k}$ and the loss estimation of RINLF is more consistent with the failure rate than that of the revised Taguchi loss function and INLF, it is suggested that RINLF be used in the determination of economic specification limits.

\section{SUMMARY}

In the manufacturing process of an industrial product, the determination of engineering specification and the selection of loss function have a significant impact on the estimation of quality loss. In this paper, we propose a new method for determining the economic specification limits for the manufacturing processes with symmetric or asymmetric losses. By exploring the relationship between loss functions and process capability indices for symmetric and asymmetric tolerances and then comparing the unit total expected loss, we have shown that RINLF is the most reasonable one to reflect the actual quality loss/failure rate among three loss functions. Therefore, it is suggested that RINLF be used in the determination of new economic specification limits.

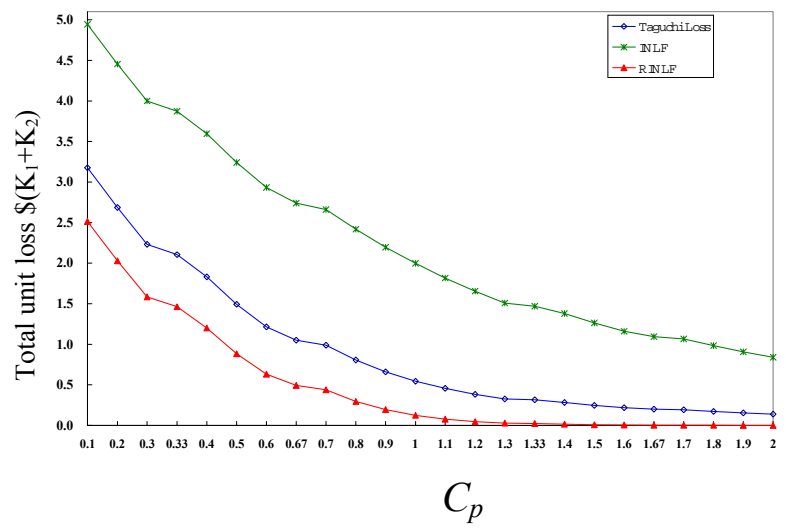

Fig. 2. Comparison of the total expected losses per unit product for various loss functions under different process capability indices for the symmetric tolerance and symmetric loss case

\section{ACKNOWLEDGMENT}

The first author would like to gratefully acknowledge financial support from the National Science Council of Taiwan on this research project.

\section{REFERENCES}

[1] K.C. Kapur and C.J. Wang,“Economic Design of Specifications Based on Taguchi's Concept of Quality Loss Function," Quality Design, Planning, and Control, The American Society of Mechanical Engineers, Boston, pp. 23-36, 1987.

[2] K.C. Kapur, "An Approach for Development of Specifications for Quality Improvement," Quality Engineering, vol. 1, pp. 63-77, 1988.

[3] K.C. Kapur and B.R. Cho, "Economic Design of the Specification Region for Multiple Quality Characteristics, " IIE Transactions, vol. 28, pp. 237-248, 1994.

[4] R. V. Leon and C. F. Wu, "A Theory of Performance Measures in Parameter Design," Statistic Sinica, vol. 2, pp. 335 358, 1992.

[5] J. N. Pan and J. H. Wang, "A Study of Loss Functions for Product Interference Analysis", Industrial Engineering Research, vol. 2(1), pp. 80-100, 2000.

[6] T. P. Ryan, Statistical Methods for Quality Improvement, Wiley, New York, 1989.

[7] F. A. Spring, "The Reflected Normal Loss Function," The Canadian Journal of Statistics, vol 21, pp. 321-330, 1993.

[8] F. A. Spring and A. S. Yueng, "A General Class of Loss Functions with Industrial Applications, "Journal of Quality Technology, vol. 30(2), pp. 152-162, 1998.

[9] G. Taguchi, Introduction to Quality Engineering: Designing Quality Into Products and Processes, Asian Productivity Organization, Tokyo, 1986.

[10] M. Tribus and G. Szonyi, "An alternative view of the Taguchi approach," Quality Progress, vol. 22(5), pp. 46-52, 1989. 